\title{
Mobile Learning Engine Moodle (MLE - Moodle): das funcionalidades a validação em curso a distância utilizando dispositivos móveis
}

Patric da Silva Ribeiro - PPGI/UFSM/RS - patricribeiro@uabrestingaseca.com.br

Roseclea Duarte Medina - PPGI/UFSM/RS - roseclea.medina@ gmail.com

\section{Resumo}

Esse artigo tem como principal objetivo apresentar e analisar os recursos oferecidos pelo Ambiente Virtual de Aprendizagem Móvel (AVAM) - Mobile Learning Engine Moodle (MLE - Moodle), durante a capacitação sobre a Linguagem de Programação HTML aos acadêmicos do Curso de Especialização em Tecnologias da Informação e da Comunicação Aplicadas a Educação da Universidade Federal de Santa Maria (UFSM), elencando as ferramentas e suas possibilidades de utilização. Aplicou-se a Análise de Questionários e Observação, a fim de avaliar a utilização de dispositivos móveis no processo de ensino aprendizagem.

Palavras - Chave: MLE-Moodle, m-learning, dispositivos móveis, avaliação.

\section{Mobile Learning Engine Moodle (MLE - Moodle): features validation of the current distance using a mobile device}

\begin{abstract}
This article has as main objective to present and analyze the resources offered by the Virtual Environment for Mobile Learning (AVAM) - Moodle Mobile Learning Engine (MLE - Moodle) during training on the HTML programming language to students of the Course of Specialization in Technology Applied Information and Communication Education, Federal University of Santa Maria (UFSM), listing the tools and their potential use. Applied to analysis of questionnaires and observation to assess the use of mobile devices in the process of teaching learning.
\end{abstract}

Keywords: MLE-Moodle, m-learning, mobile devices, evaluation.

\section{Introdução}

Nas últimas décadas, têm-se vivenciado o surgimento de uma sociedade móvel e conectada, com uma variedade de fontes de informação e modos de comunicação disponível. Em concordânica com os autores Tarouco e Meirelles (2005) citada por Naismith "et al". (2004),

Tecnologias móveis estão sendo incorporadas de forma ubíqua e em rede, permitindo interações sociais relevantes, sensíveis ao contexto e possibilitando conectividade com a Internet. Tais tecnologias podem ter um grande impacto na aprendizagem.

Os estudantes poderão mover-se cada vez mais para fora da sala de aula em direção a um ambiente de aprendizagem, real ou virtual, caracterizado por uma aprendizagem contextualizada, personalizada e colaborativa. Neste contexto, o surgimento de novas tecnologias aumenta a complexidade dos ambientes virtuais de aprendizagem, por isso, estes necessitam adaptar-se a uma computação altamente dinâmica, onde o ambiente está em constante mudança em função da mobilidade do usuário.

De acordo com os autores Ogata (2004) e Rogers (2005), 
Os ambientes virtuais de aprendizagem precisam evoluir para este novo modelo, suportando processos educacionais em qualquer lugar e tempo, tanto virtual, quanto real do aprendiz.

O tema de pesquisa deste artigo apresentou a utilização dos dispositivos móveis na educação, e está centrado nas possibilidades de impacto de seu uso no processo de ensino e aprendizagem, não no acesso propriamente dito, mas na incorporação dessa tecnologia como ferramenta para ensinar e aprender. Analisando estes aspectos e os trabalhos em desenvolvimento na área, conclui-se que os ambientes virtuais de aprendizagem, face ao cenário da computação atual, precisam evoluir no suporte à mobilidade, tanto das aplicações quanto dos aprendizes.

Tendo em vista a importância deste novo cenário educacional aberto, dinâmico e adaptável, permitindo que processos ocorram em qualquer tempo e lugar, de forma contínua, contextualizada e integrada ao cotidiano do aprendiz, é que foi elaborado este artigo. A necessidade de aprofundamento no tema m-learning, definiu os objetivos deste trabalho, que foi avaliar os recursos oferecidos pelo AVAM MLE-Moodle (Ambiente de Aprendizagem Móvel MLE-Moodle) durante a capacitação sobre a Linguagem de Programação HTML realizada por acadêmicos do Curso de especialização em Tecnologias da Informação e da Comunicação Aplicadas a Educação da Universidade Federal de Santa Maria.

O presente artigo constitui-se de seis seções. A seção dois apresenta AVAMs. A seção três é dedicada à Avaliação de AVAs. A metodologia é apresentada na seção quatro. Os resultados e discussões são descritos na seção cinco. A seção seis é composta das considerações finais e finalmente são apresentadas as referências.

\section{Ambientes Virtuais de Aprendizagem Móveis (AVAMs)}

O processo de ensino - aprendizagem deve ser concebida de forma a permitir uma perfeita integração entre as ferramentas tecnológicas e as ações de aprendizagem utilizadas na educação, e com o avanço das tecnologias de informação e comunicação têm estimulado o desenvolvimento das mais diversas e inovadoras formas de difundir conhecimento através da World Wide Web. Conseqüência dessa evolução, a educação a distância tomou um novo impulso que favoreceu a disseminação e a democratização do acesso à educação em diferentes níveis e formas de interação e aprendizagens. Neste contexto, discute-se hoje o conceito de Aprendizagem com Mobilidade - Mobile Learning ou M-Learning definida como:

processos de aprendizagem que ocorrem, necessariamente apoiados pelo uso de TIMS (tecnologias de informação móveis) e que tem como característica fundamental a mobilidade de atores humanos, que podem estar fisicamente/geograficamente distante de outros atores e também de espaços físicos formais de educação, tais como salas de aula, salas de treinamento ou local de trabalho (Bowker, 2000; Koschembahr, 2005).

Seu grande potencial encontra-se na utilização da tecnologia móvel como parte de um modelo de aprendizado integrado, caracterizado pelo uso de dispositivos de comunicação sem fio, de forma transparente e com alto grau de mobilidade (Ahonen, 2003).

O M-Learning é a fusão de diversas tecnologias de processamento e comunicação de dados que permite a estudantes e professores uma maior interação. Sintetizando, M-learning é uma nova forma de interação por meio de dispositivos móveis. Segundo Silva e Consolo (2004) citada por Mendes "et al". (2006), o Mobile Learning é um desenvolvimento do e-learning, ou aprendizagem por meios eletrônicos. 
Devido a crescente utilização de dispositivos móveis, aplicações que eram conhecidas apenas em ambiente desktops, passaram a ser disponibilizadas neste novo tipo de plataforma. Além de aplicações tornou-se possível também o pleno acesso a Internet, facilitando ainda mais o alcance aos mais variados tipos de informações.

Nesse sentido, tornou-se possível o surgimento de novos ambientes de programação focados neste tipo de equipamento, onde é possível desenvolver aplicações quase que totalmente independentes de dispositivo fabricante, com as plataformas Flash lite, Brew, Android e J2ME que propiciam o M-Learning surgir em ambientes como: CULE (Yang, 2006), CLUE (Ogata, 2004), GlobalEdu (Barbosa et al., 2008) MLEMoodle (MLE-Moodle - End users, 2009) entre outros. A seguir é apresentado o ambiente MLE - Moodle e suas funcionalidades.

\subsection{Mobile Learning Engine Moodle (MLE - Moodle)}

Nos últimos quatro anos a Universidade de Ciências Aplicadas Joanneum, Áustria, no Curso de Pós-Graduação em Gestão da Informação, Departamento de Tecnologias e Mídias Digitais, teve várias teses e projetos relacionados com a "aprendizagem móvel" (Mobileclasrrom, 2009).

A partir de alguns trabalhos como: a tese "Mobile Classroon" de Andreas Fortsting (2006), Mobile Learning Solutions MLE (Mobile Learning Engine) (2004) e a tese "Mobile Learning Motor", de Matthias Meisenberger, que teve como objetivo adaptar a interface do Moodle para dispositivo móvel, e o trabalho Moodle Mobile (MOMO) que criou uma base para integração das configurações, usuários, cursos e conteúdo do Moodle com desenvolvimento de um aplicativo com o objetivo reduzir o tempo de conexão do aluno a rede com o celular para realizar as atividades, permitiu que todas essas soluções combinadas desse inicio ao Projeto MLE-Moodle que tornou possível acessar o conteúdo criado diretamente no Moodle por meio dos dispositivos móveis (Yingling, 2006).

MLE-Moodle (Mobile Learning Engine - Moodle) é de código-fonte livre, totalmente gratuito e personalizável, vinculado ao Ambiente Virtual de Aprendizagem (AVA) Moodle. As especificações podem ser adaptadas conforme necessário com WML, PHP e MySQL. Quaisquer alterações efetuadas ao Moodle são automaticamente convertidas para os dispositivos móveis também (Yingling, 2006).

$\mathrm{O}$ acesso do MLE-Moodle pelo celular é realizado por meio do navegador do dispositivo ou pode-se usar um módulo especial para auxiliar no processo de aprendizagem com dispositivos móveis, MLE-Cliente (Meisenberger, 2004).

O Mobile Learning Engine permite que o aluno aprenda quando e onde quiser, mas se estiver em casa ou na escola, na frente de seu PC / laptop não faria qualquer sentido usar um celular para a aprendizagem. Por isso que o MLE é integrado com um sistema de e-learning (neste caso Moodle). Com o seu PC / laptop utiliza-se o padrão do sistema e-learning, se estiver longe do seu computador pode continuar a aprender com o seu celular.

O site do Projeto MLE-Moodle para usuários, explica que o sistema está disponível em dois idiomas: inglês e alemão, sendo as seguintes ferramentas acessadas pelo celular e que estão no e-Learning Moodle:

Lição: Consiste de um conjunto de páginas, essas normalmente terminam com uma questão ou alternativas de resposta. Dependendo da resposta escolhida pelo aluno ele prossegue na lição ou pode retornar para a mesma página. A navegação pode ser 
linear (da primeira à última) ou assumir uma estrutura bastante complexa que é executada sobre a MLE (MLE-Moodle - End users, 2009).

Quiz: É um teste constituído a partir de múltipla escolha, verdadeiro ou falso, e perguntas de respondas curtas. Cada tentativa é automaticamente marcada, e o professor pode escolher se quer dar retorno ou mostrar respostas corretas (MLE-Moodle - End users, 2009).

Questionário: Uma série de instrumentos de estudo, questões, para recompensar os alunos por terem feito a leitura recomendada e permiti verificar se eles entenderam o material lido (MLE-Moodle - End users, 2009).

Fórum: São acessíveis através do celular, pode-se ler e criar ou editar os debates e respostas. Além disso, permite anexar fotos, vídeos ou áudios para a sua discussão (MLE-Moodle - End users, 2009). Pode-se também gravar instantaneamente com o celular ou procurar no sistema de arquivos e enviar.

Wikis: Permite a construção de documentos de forma coletiva (MLE-Moodle End users, 2009).

Banco de dados: O MLE-Moodle é personalizado possuindo um banco de dados que pode ser pesquisado e exibido (MLE-Moodle - End users, 2009).

Recursos: Pode-se acessar uma variedade de recursos do Moodle por meio do celular, como: texto, texto HTML, imagens, vídeo, áudio, links e gerenciar os arquivos (MLE-Moodle - End users, 2009).

Mensagens instantâneas: MLE - Moodle integra plenamente o sistema de mensagens do Moodle. O usuário é notificado, se uma nova mensagem for enviada, não importando se foi criada através de um celular ou de um PC, portanto, não havendo necessidade de notificação por SMS (MLE-Moodle - End users, 2009).

O ambiente Moodle apresenta o bloco "Utilizadores Mobile Online", mostra os usuários da Web que estão on-line via celular. Além disso, possui uma lista de contato do MLE, permitindo iniciar chamadas telefônicas, caso o usuário tenha fornecido o seu número de telefone.

O MLE-Moodle apresenta recursos parcialmente específicos para m-Learning: Flashcard Trainer, Mobile Learning Objects (objetos de aprendizagem off-line), Mobile Tags (serviços baseados na localização) e Comunidade Móvel.

Flashcard Trainer: É uma característica totalmente nova para o Moodle que pode ser usado com o celular (m-Learning) e com o PC (e-Learning), permitindo a sincronização. Característica essa que aceita a aprendizagem continua em ambos os dispositivos. Escreve-se uma pergunta e uma resposta que fica organizado em grupo de questões, pode ter um vocabulário, data históricas, fórmulas ou qualquer assunto que pode ser aprendido por meio do formato pergunta e reposta. $\mathrm{O}$ estudante tenta recordar a solução da questão, se tiver êxito, é enviado o para o próximo grupo, questões com outro nível de dificuldade. Mas se errar, ele volta para o primeiro grupo e obriga-se em rever as questões (MLE-Moodle - End users, 2009).

Mobile Learning Objects (MLOs): É um objeto especial para a aprendizagem no MLE, pode ser armazenado no celular e posteriormente utilizado sem qualquer conexão à Internet (off-line). A aprendizagem MLOs implementa todas as funcionalidades do MLE, incluindo: interatividade entre perguntas instantâneas com correção automática, responder quizes, questionários de simples e múltipla escolha, Flashcard Trainer, entre outros (MLE-Moodle - End users, 2009). 
MLOs podem ser criados com o MLE-Editor (What-You-See-Is-Waht-You-Get), um editor para criar conteúdos. Os MLOs podem ser visualizados também com PC, portanto pode-se usar este conteúdo para ambos os casos.

Mobile Tags: Com marcação móveis criar-se facilmente etiquetas que podem ser interpretados pelo MLE (se o celular atende aos requisitos)(MLE-Moodle - End users, 2009) permitindo que diferentes tipos de etiquetas possam ser criadas, como uma etiqueta com ligação a uma URL, ou com tags específicas do Moodle para transmitir um determinado curso ou atividade diretamente para o dispositivo móvel. É ainda possível atribuir coordenadas de uma tag que pode ser usado para emular posicionamento GPS, quando está disponível. (MLE-Moodle - End users, 2009).

Mobile Comunidade: é disponibilizada a todos que utilizam MLE-Moodle (o estudante, quando é inscrito para um curso específico passa a ter acesso). Pode ser utilizados para qualquer tipo de comunidade relacionada ao curso, assim como com conteúdos especiais, calendários ou endereços de bibliotecas, que se deseja acessar facilmente (MLE-Moodle - End users, 2009).

\section{Avaliação de Ambientes Virtuais de Aprendizagem}

A avaliação de AVAs pode tomar como base para sua investigação, as condições em que a aprendizagem se realiza (estrutura), os modos pelos quais os estudantes são capazes de interagir sendo apoiados nas suas atividades (processos) e o alcance dos objetivos e das metas propostas (resultados) (Laguardia, 2007).

Os sistemas de avaliação de interfaces em AVAs são componentes fundamentais e permitem dar feedback ao administrador sobre os aspectos de usabilidade, ergonomia, confiabilidade, acessibilidade e interação do usuário com o sistema. A interface destes sistemas deve ser amigável e intuitiva, bem como, facilitar o seu uso e diminuir o processo exaustivo da busca de acesso a informação pelo usuário. Avaliar AVAs é uma tarefa complexa, pois além de estarem em constantes estudos e evolução, contemplam variáveis de tecnologia e de aprendizagem (Laguardia, 2007).

Para a autora Oliveira (2001),

"A acessibilidade e facilidade de uso em um sistema podem ser considerados como os fatores determinantes para a utilização ou não de um serviço de informação. Sendo assim, requer constante feedback para que esses serviços possam ser planejados e atendam as necessidades presentes e continuadas dos seus usuários".

\subsection{Métodos de avaliação de Ambientes Virtuais de Aprendizagem}

Benigno e Trentin (2000), afirmam que:

"Na avaliação de AVAs, é necessário dispor de dados sobre características individuais dos participantes (observação do comportamento), do ambiente de aprendizagem, participação, comunicação, materiais e da tecnologia utilizada."

Foram utilizados os seguintes métodos de avaliação: observação e questionários, apresentados a seguir.

Dixon (2001) afirma, “A aplicação de questionários pode ser realizada de forma presencial ou on-line, apresentando as seguintes vantagens: rapidez na coleta dos dados, uso de grandes amostras, menor custo de administração e processamento e taxas de retorno mais altas". 
A observação dos usuários pode ser de duas formas, direta ou indireta. Na forma direta o avaliador observa o comportamento do usuário, como seqüência de ações. $\mathrm{O}$ usuário pode modificar o comportamento pelo fato de estarem sendo observado. $\mathrm{Na}$ observação indireta são coletadas informações de forma automática, não interferindo no comportamento do usuário.

Para o presente trabalho, as abordagens de avaliação foram: observação e questionário.

\section{Metodologia}

Com a finalidade de gerar conhecimentos para aplicação prática, dirigida à solução de problemas específicos, foi desenvolvida uma pesquisa aplicada, objetivando integrar o estudo sobre a importância da ergonomia e usabilidade.

Este levantamento é parte integrante da dissertação de Mestrado sobre o AVAM Mle-Moodle que surgiu da necessidade de conhecer e de observar a aplicabilidade do mesmo, analisando a reação dos alunos, elencando as ferramentas e observando as possíveis melhorias, com o intuito de após implementar ao ambiente um repositório de vídeos embasado nas tecnologias que compõem a web semântica permitindo o acesso via dispositivo móvel e desktop para apoiar no processo educacional.

Nos tópicos seguintes serão apresentadas as descrições do experimento, caracterizações da população entrevistada, processo de desenvolvimento e acompanhamento.

\subsection{Descrição do experimento}

A metodologia experimental deste trabalho selecionou vinte acadêmicos do Curso de Especialização em Tecnologias da Informação e da Comunicação Aplicadas a Educação da Universidade Federal de Santa Maria (UFSM), que estavam realizando o Curso de Capacitação - Linguagem de Programação HTML, promovido pelo Pólo Educacional Superior de Restinga Sêca/RS.

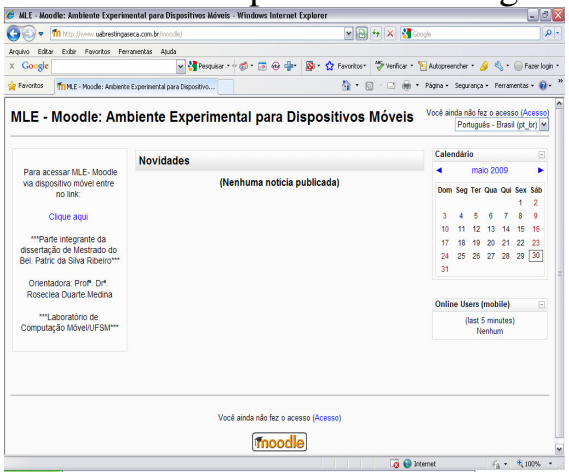

Figura 1 - Plataforma Moodle

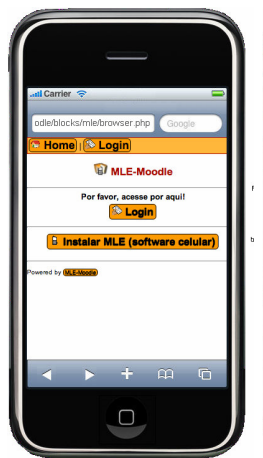

Figura 2 -Plataforma MLE-Moodle

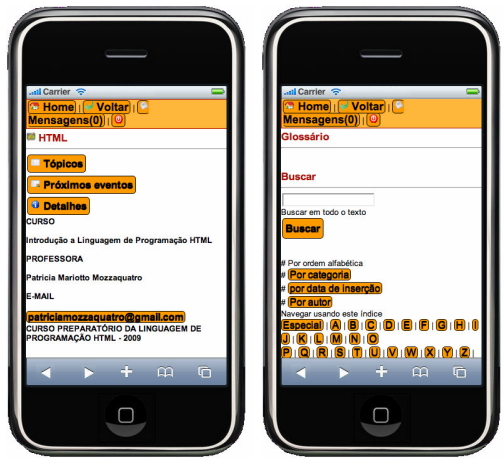

to, o processo foi desenvolvido nas seguintes etapas: a) Foi instalado o AVAM Moodle 1.9; b) Logo após os módulos do Mle-Moodle (Versão 0.79), cuja interface para PC foi configura da para ser acessada no endereço http://www.uabrestingaseca.com.br/moodle (Figura 1) e no navegador do celular pelo endereço http://www.uabrestingaseca.com.br/ moodle/blocks/mle/browser.php conforme a Figura 2, intitulou-se como Ambiente 
Experimental para Testes com Dispositivos Móveis; c) Foi realizada a tradução da versão em inglês para Português-Brasil; d) Preparou-se o ambiente com as atividades de ensino-aprendizagem do Curso de Linguagem de Programação HTML; e) Elaboração do questionário de avaliação embasado nos critérios citados na Seção 3; f) Interação com o Ambiente Virtual de Aprendizagem via Laptop/PC e via celular e smartphone; g) Aplicação do Questionário Avaliativo; h) Análise quantitativa dos resultados; i) Apresentação dos resultados obtidos e Elaboração das conclusões.

A avaliação foi feita da seguinte forma: os usuários testaram o sistema com a utilização de um Iphone 3G (SO 2.1) e um celular Nokia 2630, modelos disponíveis para essa pesquisa, seguindo um roteiro de funções pré-definidas, onde eles deveriam cumprir algumas etapas. Após estas interações com a interface o mesmo deveria responder os itens do questionário, de acordo com a sua percepção no momento dos testes.

Enquanto o usuário realizava os testes, nos casos que era possível observaram-se as reações do usuário e anotavam-se possíveis melhorias, tomando como base também as dificuldades notadas.

Com as informações obtidas através destas duas entradas gerou as conclusões descritas na próxima seção.

\section{Resultados e Discussão}

Os resultados decorreram da compilação do formulário aplicado conforme demonstrados a seguir, levando em consideração questões pontuais como ferramentas de autoria, trabalho coletivo, perspectivas didático-pedagógicas, interface, cooperação, ferramentas síncronas e assíncronas, adequadação e usabilidade, e outras questões dissertativas.

Os alunos da pesquisa encontram-se na faixa etária de vinte um a quarenta anos, e mais de $75 \%$ são do sexo feminino.

Observou que todas as atualizações realizadas no Moodle eram refletidas no MLE-Moodle, porém o acesso via dispositivo móvel, neste caso, smartphone e celular, oferece somente a visão de aluno e não de professor, neste caso, as ferramentas de autoria são acessadas somente via e-learning.

A ferramenta de trabalho coletivo Wiki gerou alguns comentários negativos relacionados ao espaço disponível para edição de textos, o mesmo se apresenta na forma de uma caixa de texto estreita não dando uma visão geral de um texto e sim de uma frase no Nokia 2630, e no Iphone $3 \mathrm{G}$ a caixa de edição do texto não fica habilitada, e o aluno não pode digitar o texto, somente visualizar.

Em relação às ferramentas da plataforma $M L E$-Moodle que foram de fácil aprendizagem e navegação temos o Fórum com $45 \%$, seguido do questionário com $24 \%$, conforme ilustrado na figura 3 .
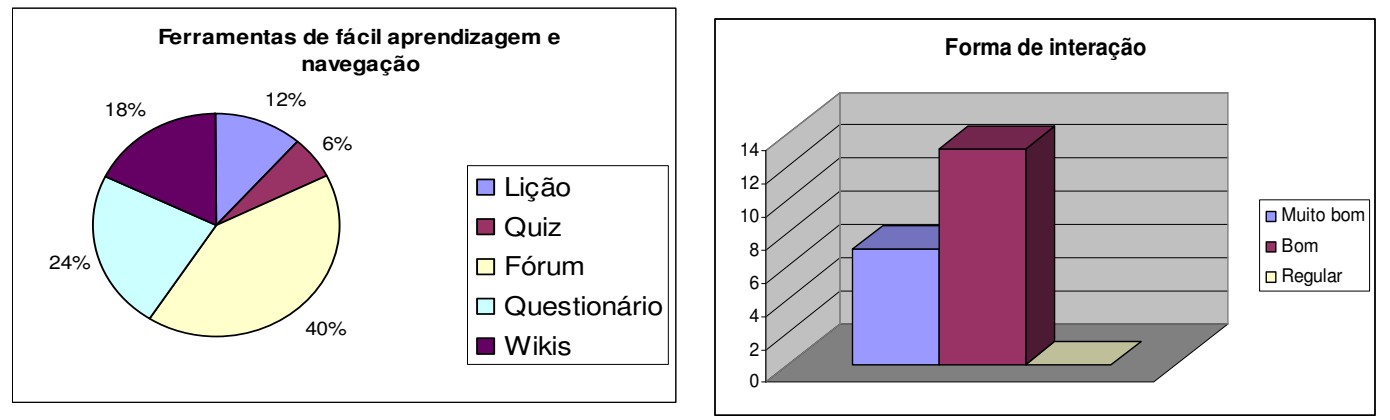
Figura 3 - Ferramentas de fácil aprendizagem e navegação e Figura 4 - Forma de interação geral.

Constatou-se que o ambiente oferece apenas ferramentas de interação assíncrona sendo as mais utilizadas pelos alunos foi o fórum (55\%) e em segundo as mensagens $(45 \%)$. Observou-se que também a ferramenta que mais gostaram de utilizar via dispositivo móvel foi o fórum, segundo eles pela possibilidade de anexar imagens ou áudio, sendo semelhante ao recurso de SMS que é de conhecimento dos mesmos, apesar de que no Iphone $3 \mathrm{G}$ o recurso de anexar arquivos não funcionou no dispositivo.

Em relação à interface com o usuário apresentada, constatou-se que, quanto às cores e sua disposição na tela dos dispositivos, a tela inicial do ambiente e ferramentas foi classificada como boa. Quanto ao tipo de fonte utilizada, também foi considerada adequada apresentando os menus de forma clara. A navegação pelo sistema e envio de tarefas enquadram-se na categoria bom, pois as telas proporcionam uma navegação de forma rápida e agradável.

Alguns alunos utilizaram os dois dispositivos, os que trabalharam com o dispositivo Nokia 2630 e depois com o Iphone 3G sentiram diferença, na questão da navegação, onde $90 \%$ achou mais fácil utilizar o segundo dispositivo devido ao recurso da tela ser sensível ao toque e de tamanho maior e $10 \%$ acharam complicado o layout do aparelho preferindo o Nokia 2630.

$\mathrm{O}$ design da interface na forma de um todo foi considerado bom. $\mathrm{O}$ aspecto relacionado ao desempenho do ambiente a maioria dos entrevistados consideraram

Em relação ao formato dos materiais, tanto audiovisuais, quanto os gráficos ou desenhos, observou-se que depende do tipo de aparelho ter os recursos habilitados, no dispositivo Nokia 2630 a visualização dos documentos (doc, xls, ppt, pdf) ficou prejudicado em virtude do tamanho da tela. No Iphone $3 \mathrm{G}$ os documentos foram visualizados normalmente, porém certas imagens inclusas no ambiente (342 x 295 pixels), ficaram desproporcionais para o tamanho da tela para os dois modelos de celulares, sendo necessário utilizar as barras de rolagem do browser, lembrando da necessidade do professor ter um cuidado a esses detalhes.

Quando questionados em relação à forma de interação geral oferecida pelo ambiente MLE-Moodle pelo dispositivo móvel a maioria classificou como boa, figura

Para melhor embasar a pesquisa foram feitos questionamentos aos acadêmicos: Qual das ferramentas que acessada via dispositivo móvel dificultou a sua realização? "O wiki é difícil de ler e analisar o texto para poder trabalhar coletivamente, faz falta as ferramentas de formatação do texto" (Aluno A). "Achei meio confusa a ferramenta FlashCard Trainer" (Aluno B).

Sobre a ferramenta nova FlashCard Trainer não conseguiram compreender no primeiro momento, mostrando que a tradução para o português, não ficou clara, sendo um ponto a ser melhor interpretado e traduzido.

Outro aspecto abordado foi o grau de satisfação ou insastifação em ter acesso ao conteúdo de aprendizagem com MLE-Moodle. Os alunos expõem o seguinte: "Eu adorei esse recurso, imagina quando sobra um tempinho livre em qualquer lugar poderia ver as atividades que estão sendo postadas no ambiente e inclusive realizar algumas atividades, não ficando na dependência sempre de um PC" (Aluno B); "Eu não tenho computador em casa, mas tenho celular isso me ajudaria muito, pois poderia acessar o ambiente se tivesse algo novo, iria para o Polo, e com isso poderia aproveitar melhor meu tempo livre"(Aluno C). "Gostei, mas achei ruim de ler o material tenho problema de visão é tão pequeno estas telas, e não tenho pratica nesses coisas, usaria muito pouco" (Aluno D). 
Como a turma é formada por professores perguntamos a eles se utilizariam o ambiente MLE-Moodle para auxiliar em suas atividades educacionais? "Sim utilizaria, pois vem para contribuir para o processo aprendizagem oferecendo outra forma de acesso, mas depende do conhecimento que o indivíduo possui sobre seu funcionamento e sobre o dispositivo móvel" (Aluno D); "Utilizaria sim, é muito bom, os cursos a distância tem que oferecer autonomia aos alunos e com esse sistema ele poderia ir a busca do conhecimento sem estar preso a um PC, um laboratório, polo ou lan house." (Aluno E).

\section{Considerações Finais}

O instrumento de pesquisa mostrou-se válido e viável, pois através do mesmo, foi realizada a análise e avaliação AVAM MLE-Moodle, utilizado no Curso de Capacitação Linguagem de Programação $H T M L$, que possibilitou a percepção de como acontece à funcionalidade do ambiente, vislumbrando possíveis melhorias amparadas nas opiniões colocadas.

As pesquisas mostraram uma satisfação por parte dos alunos que experimentaram o ambiente que se mostrou um estimulante para o processo de aprendizagem, porque além de ser uma novidade, eles perceberam uma grande utilidade na sua vida diária. E com isso, há uma abertura maior de possibilidades de atividades que podem ser realizada com a utilização dos dispositivos móveis.

As avaliações realizadas surtiram efeito positivo, cumprindo assim o objetivo de conhecer, e ver a usabilidade dentro do contexto do EaD, para possíveis melhorias e inclusão de um repositório de vídeos com base nas técnicas de web semântica para trabalhos futuros.

Outra oportunidade que surge é a de testar o sistema com um público muito maior e uma variedade ampla de dispositivos móveis.

\section{Referências}

BARBOSA, D. N. F. et al. GlobalEdu - an architecture to support learning in a Pervasive Computing Environment. 2005. In: IFIP TC10 Working Conference: EduTech 2005. Perth. New York : Springer, 2005.

BENIGNO, V.; TRENTIN, G. The evaluation of online courses. Journal of Computer Assisted Learning, v. 16, p. 56-70, 2000.

BOWKER, R.R. Wireless Trainning or "m-learning" is here: first movers in the pool. Lifelong learning. Market report, ( p. 5-22), 2000.

DIXON, J. Evaluation tools for flexible delivery (workshop version). Melbourne: TAFE frontiers, 2001.

FORSTINGER, Andreas. Mobile Classrom. Resumo da Dissertação Universidade de Ciências Aplicadas JOANNEUM. Disponível em: <http://www.fhjoanneum.at/aw/home/Studienangebot/fachbereich_information_design_technologien/i ma/Menschen/AbsolventInnen/ bbqe/Abschlussarbeiten-Details_ima/?arbid=4295733 051\&arbtyp=Dipl.-Arbeit\&lan=en $>$ Acesso em: 15 abr. 2009. 
KOSCHEMBAHR, Christopher Von. Mobile Learning: the next evolution. Chief Learning Officer, fev, 2005.

LAGUARDIA, J. et. al., 2007. Avaliação em ambientes virtuais de aprendizagem. Educação e Pesquisa. São Paulo, 2007

MEIRELLES, Luiz Fernando T.; TAROUCO, Liane M. R. Framework para Aprendizagem com Mobilidade. Universidade Federal do Rio Grande do Sul (UFRGS), 2005. Disponível em: < http://ead.ucpel.tche.br/portal/publicacoes/pdf/artigosbie2005-final.pdf>. Acesso em: nov. 2008.

MEISENBERGER, M. MLE - Mobile Learning Engine. Dissertação Universidade de Ciências Aplicadas JOANNEUM. 2004. Disponível em: <http://drei.fhjoanneum.at/mle/docs/diplomarbeit_mLearn_2004_05_28.pdf >. Acesso em: 5 abr. 2009.

MLE-MOODLE - END USERS. MLE-Moodle. 2009. Disponível em: <http://mle.sourceforge.net/ mlemoodle/index.php?lang=en>. Acesso em: 16 abr. 2009.

MOBILE CLASSROOM. 2009. Disponível em: <http://moodle.mobileclassroom.at /moodle18/index.php?lang=en_utf8>. Acesso em: 15 abr. 2009.

NAISMITH, L.; LONSDALE, P.; VAVOULA, G.; Shet Sharples, M. S. REPORT 11: Literature Review in Mobile Technologies and Learning. NESTA FUTURELAB SERIES, University of Birmingham. 2004.

OLIVEIRA, Elaine Rosangela de. (2001). Avaliação Ergonômica de Interfaces da Scielo - Scientific Electronic Library Online. Disponível em: < http://teses.eps.ufsc.br/defesa/pdf/4705.pdf>. Acesso em: 10 set. 2008.

OGATA, H., Yano, Y. Knowledge awareness for a computer-assisted language learning using handhelds. International Journal of Continuous Engineering Education and Lifelong Learning, v. 14, n. 4-5. Jan, 2004.

ROGERS, Y.; et al. Ubi-learning Integrates Indoor and Outdoor Experiences. ACM Communications, v. 48, n. 1, Jan. 2005. p.55-59.

SILVA, Maria da Graça Moreira da; CONSOLO, Adriane Treinero. Uso de dispositivos móveis na educação - o SMS como auxiliar na mediação pedagógica de curso a distância. Disponível em: < http://www.5e.com.br/info design/146/Dispositivos _moveis.pdf>. Acesso em: nov 2008.

SYVÄNEN, A.; AHONEN, M.; JÄPPINEN, A.; PEHKONEN, M.; VAINIO, T. Accessibility And Mobile Learning. In: IFIP ETRAIN CONFERENCE IN PORI, Finland, 2003.

YINGLING, M. Mobile Moodle. Muhlenberg College. Disponível em: < http://portal.acm.org/ citation.cfm?id=1127487>. Acesso em: 18 mar. 2009.

YANG, S. J. H. Context Aware Ubiquitous Learning Environments for Peer-to-Peer Collaborative Learning. 2006. Educational Technology \& Society, 9 (1), 188-201. 
